\title{
Philosophiques
}

\section{Husserl et Stumpf sur la Gestalt et la fusion}

\section{Carlo Ierna}

Volume 36, numéro 2, automne 2009

Edmund Husserl (1859-1938)

URI : https://id.erudit.org/iderudit/039482ar

DOI : https://doi.org/10.7202/039482ar

Aller au sommaire du numéro

Éditeur(s)

Société de philosophie du Québec

ISSN

0316-2923 (imprimé)

1492-1391 (numérique)

Découvrir la revue

Citer cet article

Ierna, C. (2009). Husserl et Stumpf sur la Gestalt et la fusion. Philosophiques, 36(2), 489-510. https://doi.org/10.7202/039482ar

\section{Résumé de l'article}

Dans la seconde édition des Logische Untersuchungen, Husserl affirme avoir investigué le premier, dans l'école de Brentano, les objets d'ordre supérieur et les qualités de forme. En effet, on retrouve dans Philosophie der Arithmetik une discussion des moments figuraux et de la fusion qui pourrait donner un certain appui à une telle affirmation. En considérant les concepts de Gestalt et de fusion dans leur contexte historique - particulièrement en relation à Stumpf en ce qui concerne ce dernier concept - nous constatons que Husserl a effectivement une conception plutôt originale et intéressante de ces phénomènes d'ordre supérieur. 


\title{
Husserl et Stumpf sur la Gestalt et la fusion
}

\author{
CARLO IERNA \\ Husserl-Archives, Leuven \\ carlo.ierna@hiw.kuleuven.be
}

\begin{abstract}
RÉSUMÉ. - Dans la seconde édition des Logische Untersuchungen, Husserl affirme avoir investigué le premier, dans l'école de Brentano, les objets d'ordre supérieur et les qualités de forme. En effet, on retrouve dans Philosophie der Arithmetik une discussion des moments figuraux et de la fusion qui pourrait donner un certain appui à une telle affirmation. En considérant les concepts de Gestalt et de fusion dans leur contexte historique - particulièrement en relation à Stumpf en ce qui concerne ce dernier concept - nous constatons que Husserl a effectivement une conception plutôt originale et intéressante de ces phénomènes d'ordre supérieur.
\end{abstract}

\begin{abstract}
In the second edition of the Logische Untersuchungen Husserl claims to have investigated higher order objects and Gestalt qualities before anyone else in the School of Brentano. Indeed, in the Philosophie der Arithmetik we find a discussion of figural moments and fusion that could lend some support to such a claim. By considering the concepts of Gestalt and Verschmelzung in their relevant historical context, the latter especially in connection to Stumpf, we find that Husserl indeed gave a quite original and interesting account of such higher order phenomena.
\end{abstract}

L'influence philosophique de Carl Stumpf sur le développement du jeune Husserl est secondaire peut-être uniquement en rapport avec celle de Brentano ${ }^{1}$. Husserl décida de "consacrer sa vie complètement à la philosophie » et non aux mathématiques après avoir assisté aux leçons de Brentano dans les années 1884-1886, puis, à l'automne $1886^{2}$, il se rendit à Halle pour y travailler à son Habilitationsschrift sous la supervision de Carl Stumpf, un ancien étudiant de Brentano. Sous la supervision de Stumpf, Husserl réorienta son projet sur «le problème de la continuité ${ }^{3}$ vers "des recherches logiques sur les concepts fondamentaux et les principes de l'arithmétique, et de l'analyse supérieure ${ }^{4}$. Il ne fait aucun doute que les leçons de Stumpf ${ }^{5}$ ont largement contribué au développement du premier Husserl ainsi qu'à la méthode et au thème de ses premiers travaux ${ }^{6}$.

Alors que Philosophie der Arithmetik était dédié à Brentano, Logische Untersuchungen est quant à lui dédié à Stumpf, "par admiration et amitié ».

1. Voir les chapitres pertinents de Rollinger, 1999.

2. Voir Schuhmann, 1977, p. 13, et Gerlach et Sepp, 1994, p. 165.

3. Schuhmann, 1977, p. 17.

4. Husserl, 1994, vol. I, p. 5. Voir aussi Ierna, 2005, p. 6-8.

5. Les notes personnelles de Husserl sur les leçons de Stumpf sont conservées aux Archives Husserl: Stumpf, WS 1886-1887a; Stumpf, WS 1886-1887b: Stumpf, SS1887.

6. Voir Ierna, 2006, pp. 66-80. 
Dans la troisième recherche, Husserl y fait une affirmation d'une importance considérable, témoignant de son développement philosophique en connexion avec les autres étudiants de Brentano. Alors qu'il ne fournit que des indices subtils dans la première édition, il est plutôt explicite dans la seconde:

[Philosophie der Arithmetik], mon œuvre de jeunesse (l'élaboration de ma [...] thèse d'habilitation de 1887) est généralement à comparer avec le présent ouvrage en ce qui concerne tous les développements relatifs aux ensembles, aux moments d'unité, aux complexions, aux touts et aux objets d'ordre supérieur. Je dois exprimer mon regret sur le fait que, dans plusieurs nouveaux traités sur la théorie des "qualités de forme », cette œuvre soit restée le plus souvent ignorée, bien qu'une partie non négligeable des récents développements de Cornelius, Meinong, etc., sur les questions d'analyse, d'appréhension des pluralités et des complexions se trouvent déjà dans Philosophie der Arithmetik, bien que dans une terminologie différente. Il me semble qu'il serait encore utile aujourd'hui d'étudier Philosophie der Arithmetik pour les problèmes phénoménologiques et ontologiques correspondants, celui-ci étant le premier écrit à se pencher sur les actes et les objets d'ordre supérieur et à les étudier de manière approfondie ${ }^{7}$.

De telles affirmations, seulement partiellement surprenantes, méritent d'être citées en entier. Rétrospectivement, en 1913, Husserl décrit Philosophie der Arithmetik comme contenant d'importantes analyses phénoménologiques qui sont encore pertinentes pour sa position d'alors, réduisant du même coup l'ouvrage à une simple "élaboration" de sa thèse d'habilitation $^{8}$. Non seulement c'est là une affirmation forte quant à la continuité et la cohérence de ses positions de 1887 à 1913 - y compris les changements de position allant du psychologisme à l'antipsychologisme et du réalisme à l'idéalisme, qui ont été décrits comme des "révolutions » et des "ruptures » - mais c'est aussi une affirmation de priorité (presque) absurde eu égard au concept de Gestalt de von Ehrenfels et au développement de la Gegenstandstheorie de Meinong. Or une analyse des concepts de fusion (Verschmelzung) et de Gestalt tels qu'ils interviennent dans les propres travaux de Husserl et dans ceux de ses maîtres et collègues pourra nous apporter quelque lumière sur la vérité de ces affirmations?.

Bien que la notion de Verschmelzung chez Husserl et Stumpf ait déjà été discutée en détail ${ }^{10}$, je crois qu'il est néanmoins pertinent de l'examiner à nouveau en connexion plus étroite avec la notion de Gestalt tout aussi bien analysée jusqu'à présent ${ }^{11}$. En effet, on retrouve dans les premiers travaux

7. Husserl, 1984, p. 289.

8. Sur la relation entre la thèse d'habilitation et Philosophie der Arithmetik, voir Ierna, 2005 .

9. Sur les actes et les relations d'ordre supérieur dans les premiers travaux de Husserl, voir Ierna, 2006, pp. 72 sq.

10. Cf. entre autres Holenstein, 1972, p. 118-131 et Rollinger, 199, p. 100-114.

11. Cf. entre autres Smith, 1988a, et Mulligan, 1995. 
de Husserl une combinaison originale et fructueuse de ces deux notions. Le jeune Husserl était un philosophe très éclectique, intégrant avec succès des notions issues de différentes traditions et disciplines dans son propre cadre conceptuel. De plus, la conception husserlienne des moments figuraux dans Philosophie der Arithmetik ${ }^{12}$ ne combine pas seulement des intuitions d'Ernst Mach et de Stumpf, mais elle se situe également dans un contexte et une discussion historiques riches et bien articulés.

Le concept de Gestalt est habituellement associé à Christian von Ehrenfels et à son article séminal «Über 'Gestaltqualitäten' » ${ }^{13}$. Cette notion a été ensuite développée plus avant par les psychologues de la Gestalt de l'école de Berlin, notamment Max Wertheimer, Kurt Koffka et Wolfgang Köhler, mais aussi par l'école de Graz, notamment Stefan Witasek et Vittorio Benussi. En reculant d'un pas par rapport à cette génération de chercheurs, nous voyons que von Ehrenfels et les psychologues de Graz ont tous été étudiants d'Alexius Meinong et que les représentants de l'école de Berlin ont tous été étudiants de Carl Stumpf, ces deux maîtres ayant été des élèves de Brentano. Ainsi, la notion de Gestalt semble avoir des racines importantes dans l'école de Brentano. Cependant, aucun de ces maîtres illustres n'employa le terme de Gestalt dans un sens technique. Comme le rapporte von Ehrenfels au début de son article, son usage technique est inspiré de celui des Beiträge zur Analyse der Empfindungen de $\mathrm{Mach}^{14}$. Les développements ultérieurs de cette notion nous mènent ensuite en amont, vers la psychologie philosophique de Brentano ${ }^{15}$, l'analyse des relations de Meinong ${ }^{16}$, et vers les recherches de Stumpf sur la perception de l'espace et de la musique ${ }^{17}$. En effet, c'est Stumpf qui proposa une analyse approfondie du phénomène de la Verschmelzung en musique. Nous en arrivons ainsi au personnage principal de notre titre: Husserl. Dans un parallélisme presque complet avec Ehrenfels, Husserl était également un étudiant de Brentano, influencé par les travaux de Meinong sur les relations, et qui développa une notion technique de Gestalt inspirée par Mach en 1890. Indépendamment, et peut-être même avant von Ehrenfels, Husserl employa également le terme "Gestalt» dans un sens technique pour désigner des objets complexes d'ordre supérieur: le moment de Gestalt est ce qui nous permet d'avoir l'intuition de tout complexe d'un seul coup d'œil, c'est-à-dire ce qui nous permet d'avoir l'intuition immédiate des quantités comme quantités. Dans Philosophie der Arithmetik, Husserl les appela moments figuraux.

12. Husserl, 1891, édition critique dans Husserl, 1970, traduction anglaise dans Husserl, 2003.

13. Ehrenfels, 1890. Traduction française dans Fisette et Fréchette, 2007.

14. Mach 1886.

15. Brentano, 1874.

16. Meinong, 1882.

17. Stumpf $1873 ; 1883 ; 1890 b$. 


\section{Moments figuraux}

Dans Philosophie der Arithmetik (PA), Husserl discute des aspects logiques et psychologiques de l'énumération exhaustive d'une quantité (Menge) par dénombrement. Durant le processus de dénombrement, nous avons l'intuition de chaque membre de la quantité en lui-même, mais non pas de la quantité comme tout. Car cela dépasserait nos capacités représentationnelles: nous devrions représenter chaque élément singulier en lui-même, en tant qu'identique à lui-même, différent des autres, et simultanément connecté au tout. Ainsi, on ne peut parler de la quantité que de manière symbolique ou impropre ${ }^{18}$. Mais comment savons-nous alors si quelque chose est une quantité énumérable sans précisément la compter?

On ne peut ici qu'envisager un détour: il doit y avoir dans l'intuition des signes immédiatement saisissables de la quantité sensible à partir desquels on peut reconnaître le caractère de quantité, en ce qu'ils assurent l'opérabilité des processus décrits plus haut. [...] C'est seulement lorsqu'on peut supposer que des complexes de relation enveloppant la quantité totale - qu'elle soit complètement fusionnée en unités strictes ou seulement de manière isolée - donnant à tout le phénomène de quantité un caractère particulier et immédiatement remarquable (une qualité sensible de deuxième ordre, pour ainsi dire) que l'état des choses pourrait être différent: ces caractères quasi-qualitatifs, qui

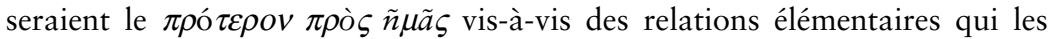
conditionnent, pourraient fournir la base respective pour l'association ${ }^{19}$.

Pour Husserl, les «quasi-qualités » d'ordre supérieur correspondraient à la forme linguistique des noms collectifs comme "essaim» (d'insectes), «banc» (de poissons), «troupeau» (de bisons), etc., qui ajoutent au pluriel simpliciter la «quasi-qualité » d'un tout unitaire, c'est-à-dire une quantité. Nous voyons directement, sans compter, que la pluralité d'objets constitue une quantité énumérable, et nous la saisissons en ce qu'elle tombe sous un seul concept collectif.

Dans chacun de ces exemples, il est question d'une quantité sensible d'objets identiques entre eux qui sont aussi nommés selon leur genre. Mais ce n'est pas tout ce qui est exprimé [...], il y a aussi une certaine propriété caractéristique de l'intuition unitaire complète de la quantité, qui peut être saisie d'un seul coup d'œil2 ${ }^{20}$.

Husserl choisit ici le terme de «moment figural» sur la base de l'analogie avec les formes spatiales. Nous voyons par exemple une similarité entre deux figures en les regardant comme un tout, et non en les regardant comme une corrélation point par point de chacun des points. De la même manière, nous voyons les quasi-qualités d'une quantité sans énumération

18. Husserl, 1891, p. 218, Husserl 1970, p. 195sq., Husserl, 2003, p. 208.

19. Husserl, 1891, p. 225; Husserl, 1970, p. 201; Husserl, 2003, pp. $213 f$.

20. Husserl, 1891, p. 228; Husserl, 1970, 203sq.; Husserl, 2003, p. 216. 
exhaustive de ses membres. Husserl mentionne explicitement «Über 'Gestaltqualitäten' » de von Ehrenfels, mais seulement pour souligner son indépendance et sa propre préséance sur ce dernier, tout en reconnaissant leur source commune, les Beiträge de Mach. En effet, Husserl prétend avoir développé sa propre théorie un an avant la parution de l'article de von Ehrenfels, c'est-à-dire en 1889.

\section{Le concept de Gestalt de Husserl}

Dans une leçon du semestre d'hiver 1889-1890 sur le concept de nombre ${ }^{21}$, Husserl emploie les termes «Gestalt» et «moment de Gestalt» en se basant sur l'œuvre de $\mathrm{Mach}^{22}$. Ce dernier a employé originellement le terme de "Gestalt» dans sa signification ordinaire afin d'indiquer un certain moment qualitatif dans les figures géométriques (les formes), mais a ensuite appliqué le terme aux mélodies dans un sens métaphorique et technique.

Lorsque nous observons deux Gestalt pareilles mais de couleur différente, par exemple deux lettres pareilles mais de couleur différente, nous reconnaissons ainsi la même forme malgré la distinction des sensations de couleur au premier coup d'œil23.

Pour Mach, les Gestalt peuvent être reconnues malgré les différences et grâce à l'égalité, c'est-à-dire la Gleichheit des éléments de la perception sensible. Ce type d'égalité se laisse remarquer "d'un seul coup d'œil », sans aucune difficulté, il est donné directement, il n'est pas déduit ou abstrait ${ }^{24}$. Mach affirmait que la similarité géométrique de deux figures complètes serait donnée par le niveau d'égalité le plus bas de leurs éléments (longueurs, angles, etc. $)^{25}$. De manière analogue à ses considérations sur l'espace, Mach applique son analyse au temps, y voyant la même perception «immédiate» de la Gestalt dans le rythme et la mélodie ${ }^{26}$. Ainsi, Mach utilisait déjà le concept de Gestalt pour indiquer la caractéristique selon laquelle un tout dépend de la configuration spécifique de ses parties. Husserl a repris cette notion et l'a appliquée à la représentation symbolique des collectiva:

Mais comment en arrive-t-on à une telle représentation symbolique? Réalisons-nous une disposition de points arbitraire au tableau ou bien imaginonsnous un nombre de points sur un cube, etc.? Qu'est-ce qui est donné en premier? Une certaine configuration de points. Il y a une intuition uniforme

21. Husserl, 2005b, p. 278-308 pair, traduction anglaise dans Husserl, 2005a, p. 279309 impair.

22. Husserl avait acheté le livre et l'avait lu immédiatement après sa parution en 1886, voir Husserl, 1891, p. 236 n.; Husserl, 1970, p. 210n; Husserl, 2003, p. 223n.

23. Voir Mach, 1886, p. 43.

24. Mach 1886, p. 45 ; cf. Mach, 1914, p. 106ssq, où Gleichheit est traduit par «identité ". Je préfère traduire ici par "égalité », pour contraster avec l'identité leibnizienne.

25. Mach 1886, p. 47; cf. Mach, 1914, p. 109.

26. Mach, 1886, p. 128; cf. Mach, 1914, p. 285. 
partant de laquelle nous pouvons remarquer ce moment de Gestalt et qui donne à tout le phénomène son aspect caractéristique. Cela constitue alors le cadre uniforme pour l'activité appréhensive: nous appréhendons un élément, allons ensuite vers un autre, puis encore vers un autre, etc. Or c'est le cadre extérieur, la Gestalt, l'uniformité de l'intuition qui nous permet de faire l'économie d'une collection véritable et qui rend possible la représentation symbolique d'une pluralité, laquelle est définie par cette intuition ${ }^{27}$.

Probablement professée en janvier 1890, cette leçon est une élaboration des thèmes de l'Habilitationsschrift de Husserl en 1887, de laquelle seul le premier chapitre fut publié tel que nous le connaissons sous le titre Über den Begriff der Zahl28. L'analyse de Husserl a été reprise dans son premier livre, Philosophie der Arithmetik, mais dans cet ouvrage Husserl a changé la terminologie de "Gestalt» pour celle de «moment figural» afin de distinguer celle-ci de l'usage de von Ehrenfels, comme nous venons de le voir. Dans le chapitre XI de la $P A$, Husserl développe le problème des intuitions symboliques de quantités plus en détail et introduit dans ce contexte la notion de moment figural pour expliquer l'appréhension des quantités en tant que quantités sans intervention de l'acte de compter. Le «moment de Gestalt » spécifique de quantités permet à Husserl d'introduire des quantités appréhendées symboliquement, et donc des nombres conçus symboliquement, ce qui constitue le thème central de la deuxième partie de $P A^{29}$. Au lieu de construire à proprement parler une quantité par un acte de colligation et par une représentation propre de son nombre basée sur l'acte de compter, on peut voir d'un seul coup d'œil qu'un certain phénomène complexe est une quantité dénombrable.

Un peu plus tard, alors qu'il rédigeait une nouvelle préface pour la seconde édition de Logische Untersuchungen, Husserl est revenu sur ce point en résumant sa position de la manière suivante:

La question de l'origine des représentations impropres de quantité me conduisit aux moments «quasi-qualitatifs ou figuraux » constitués par les «fusions » de ces relations de contenu, les mêmes que von Ehrenfels, guidé par des problèmes complètement différents, avait nommé des qualités de formes dans son traité bien connu de $1890^{30}$.

En effet, Husserl utilisera dans la troisième Recherche logique ${ }^{31}$ la notion de fusion, combinée avec la méréologie (stumpfienne), se référant

27. Husserl, 2005b, p. 298. Traduction anglaise dans Husserl, 2005a, p. 299.

28. Husserl, 1887, republié avec le sous-titre confondant «Version originale du texte jusqu'au chapitre IV» dans Husserl, 1970, p. 289-339, traduction dans Husserl, 2003, p. 305357. Pour une analyse et une comparaison de l'Habilitationsschrift perdue, de Über den Begriff der Zahl et de Philosophie der Arithmetik, voir Ierna 2005.

29. Voir aussi Hopkins, 2002.

30. «Esquisse d'une préface, second fragment », dans Husserl, 2002, p. 295.

31. Cf. entre autres Husserl, 1984, p. 240, 246-252. 
positivement à ses analyses dans $P A^{32}$ et mettant au clair le fait qu'il lui donne un sens plus large que celui de Stumpf ${ }^{33}$. Nous reviendrons un peu plus tard sur la conception de Husserl.

Le développement parallèle et indépendant de la notion philosophicotechnique de Gestalt, partagée par Husserl et von Ehrenfels, et procédant d'un arrière-plan brentanien et meinongien et de leur lecture de Mach me rend un peu moins enclin à parler d'un «tournant ${ }^{34}$ ou d'une "révolution $»^{35}$ en ce qui concerne l'article de von Ehrenfels. Étant donné le contexte de sa gestation, je dirais plutôt que l'idée était dans l'air du temps, en raison de plusieurs préoccupations et intérêts partagés. Brentano avait déjà développé une théorie de base de différents types de touts, parties, et des relations (de dépendance) entre elles, qui a été élaborée plus avant par ses étudiants Stumpf et Meinong. En effet, on trouve chez Stumpf le même genre de comparaisons qu'a fait Mach quant aux touts complexes, sur la base de la similarité non pas des contenus, mais des relations ${ }^{36}$. Il serait difficile de voir comment Husserl et von Ehrenfels auraient pu développer leurs théories sans cet arrière-plan commun et, de la même manière, il est improbable que le développement de la théorie des concepts pertinents se serait interrompu sans eux. En effet, si nous jetons un coup d'œil à la deuxième notion que j'ai mentionnée dans le titre, la fusion, on voit qu'elle est une partie d'un débat beaucoup plus large qui avait lieu indépendamment à l'époque.

\section{Fusion}

La théorie de la fusion tonale de Stumpf, basée sur la consonance, est analysée de la manière la plus détaillée dans le second volume de Tonpsychologie, mais nous allons aussi citer le premier volume ainsi que d'autres ouvrages et leçons pour clarifier certains aspects de la fusion, particulièrement en ce qui concerne les autres champs de la perception. Le terme "fusion» (Verschmelzung) n'est pas un néologisme stumpfien, mais un terme technique bien connu des travaux des autres philosophes, tels que Johann Friedrich Herbart et Wilhelm Wundt, par exemple. Stumpf a adopté le terme, mais non la théorie de ces penseurs, en lui donnant une nouvelle signification. Ainsi, il est crucial de prendre en considération ce contexte plus large dans la comparaison des usages que Stumpf et Husserl font du terme ${ }^{37}$.

32. Husserl, 1984, p. 247n.

33. Husserl, 1984, p. 249n., comme il le faisait déjà dans la PA. Husserl emploiera le terme «fusion" dans les $R l$ pour désigner également l'union intime des actes, par exemple dans l'unité de remplissement (Erfüllungseinheit), c'est-à-dire l'unité de remplissement de la signification, $c f$. Husserl, 1984, p. 44, 47, 62.

34. Smith, $1988 b$, p. 15.

35. Smith, 1994, p. 20.

36. Stumpf, 1883, p. 101.

37. Notons que Husserl a brièvement assisté aux leçons de Wundt à Leipzig alors qu'il y étudiait l'astronomie; voir Schuhmann, 1977, p. 4. 
Stumpf distingue clairement son propre usage de la fusion des autres conceptions, comme celle de Herbart: "L'expression et le concept de fusion [ne sont] aucunement en relation, tant factuelle qu'historique [...] avec la théorie psychologique générale de Herbart ${ }^{38}$.» Effectivement, Herbart avait développé une théorie de la Verschmelzung progressive infinitésimale pour rendre compte de la continuité dans la perception ${ }^{39}$. Le concept de Stumpf visait quant à lui les relations tout-parties. Ainsi, nous devons donc reconnaître avec Holenstein que la Verschmelzung de Stumpf est fondamentalement différente de celle de Herbart ${ }^{40}$, et exprimer notre désaccord avec Petitot qui affirme que c'est la même chose ${ }^{41}$. L'idée de Petitot, selon laquelle la conception de la Verschmelzung de Husserl serait proche de celle de Herbart, semble toutefois plus plausible. En effet, c'est précisément en rapport avec la similarité comme constituante pertinente de la fusion que Husserl partage un aspect important de sa théorie avec Herbart, lequel avait déjà remarqué la chose suivante à propos de l'usage de la Verschmelzung pour la constitution du concept de nombre:

Une quantité d'objets similaires est perçue. Les représentations qui en tirent leur origine se fusionnent ensemble ${ }^{42}$.

Cela ressemble à la conception husserlienne de la constitution du concept propre de nombre: à travers une série d'actes de premier ordre, nous appréhendons différents contenus, et ensuite, dans une réflexion de second ordre sur ceux-ci, nous formons un tout d'ordre supérieur, un ensemble (Inbegriff) qui a les caractéristiques d'un tout unitaire grâce à la fusion des contenus de premier ordre des relations dans les qualités, et des relations de second ordre ${ }^{43}$.

De plus, après que le multiple a commencé à se constituer par fusion en raison de ses similarités avec un concept général, ainsi que par connexion de la représentation générale avec les individus donnés, le concept de nombre est engendrét4.

Herbart indique ici la connexion entre la similarité et la fusion, en parallèle avec ce que Husserl affirmera concernant les représentations impropres des nombres. En raison de leur similarité mutuelle, la multitude de contenus primaires fusionne en une quantité (Menge) unitaire, une multiplicité dénombrable (mais non encore dénombrée). Husserl, adjoignant les positions de Herbart avec celle de Frege, critiquera la conception selon

38. Stumpf, 1890 b, p. 130 sq.

39. Cf. par exemple Herbart, 1825, p. 136-137.

40. Holenstein, 1972, p. 118.

41. Petitot, 1999, p. 338sq.

42. Herbart, 1851, vol. 6, p. 167.

43. Ierna, 2006, p. $72 s q$.

44. Herbart, 1851, vol. 3, p. 121 
laquelle le nombre serait une propriété de concept. Au lieu d'avancer que les contenus de premier ordre fusionnent en un «concept général», Husserl proposera plutôt de les considérer comme formant un ensemble (Inbegriff) ou une quantité (Menge $)^{45}$. La fusion et la Gestalt nous permettent d'appréhender des quantités en tant que quantités, mais le nombre concerne l'Inbegriff d'objet dans une quantité, et non leur concept (Begriff).

Le nombre ne se réfère donc pas au concept d'objets comptés, mais plutôt à leur ensemble ${ }^{46}$.

En partie à la suite du concept de Herbart et en le développant plus avant, Wilhelm Wundt a également employé le terme de fusion ${ }^{47}$, mais Stumpf se distancie de lui encore plus fermement:

Il est clair que le concept de fusion de Wundt [...] non seulement s'écarte du nôtre sur les points les plus essentiels, mais il n'est même pas appuyé par un seul exemple vraiment convenable ${ }^{48}$.

Cette véhémence n'est pas surprenante étant donné l'animosité entre les deux psychologues à cette époque. Dans une perspective plus large, de telles polémiques acrimonieuses entre les écoles de Brentano et de Wundt ne sont aucunement surprenantes, et ce depuis la publication concomitante de Psychologie vom empirischen Standpunkte de Brentano en 1874, et de Grunzüge der physiologischen Psychologie de Wundt ${ }^{49}$. Dans ce dernier ouvrage, Wundt caractérise la Verschmelzung de la manière suivante:

Selon des lois psychologiques générales, différentes sensations qui ont souvent été liées fusionnent ensemble de telle sorte que, dans les cas où une seule partie de celles-ci peut être réactivée par une stimulation externe ou interne, les autres s'y joignent par reproduction, sauf que les composantes reproduites possèdent généralement une intensité plus faible. Cette règle s'applique également aux organes du toucher. Sur ce plan, les sensations tactiles et les sentiments d'innervation fusionnent en des composantes inséparables ${ }^{50}$.

De plus, Wundt a également souligné que les impressions successives peuvent fusionner ensemble ${ }^{51}$. Stumpf niera à la fois que les sensations

45. Husserl reprend le terme d'Inbegriff des Paradoxien des Unendlichen de Bolzano (Bolzano, 1889), définissant l'Inbegriff comme la concaténation de "quelque chose et quelque chose et quelque chose ", etc. Comme Husserl définit le "quelque chose ", à la suite de Stumpf, comme le «concept le plus général » et ainsi le plus vide de contenu, une telle concaténation ne peut donner le "concept général» du type entendu par Herbart et Frege.

46. Husserl, 1891, p. 185; Husserl, 1970, p. 166; Husserl, 2003, p. 174. Dans l'original, la phrase au complet est en italique.

47. Voir par exemple Wundt, 1874, p. 668, n.1.

48. Stumpf, 1890 b, p. 133.

49. Brentano, 1874; Wundt, 1874.

50. Wundt, 1874, p. 483sq.

51. Ibid., p. 571. 
fusionnées soient complètement inséparables et que les sensations successives puissent fusionner de la même manière que les sensations simultanées, comme nous le verrons plus en détail plus loin. De manière encore plus explicite, Wundt a caractérisé la Verschmelzung comme une forme d'association dans sa Logik:

Nous désignons par synthèse associative une synthèse psychique [...] qui produit par fusion de sensations élémentaires des représentations composées ${ }^{52}$.

La fusion de sensations tonales appartiendrait à la classe des synthèses intensives en opposition aux synthèses extensives, car "dans la synthèse intensive, une série de sensations similaires se fusionnent ${ }^{53}$. Dans la synthèse extensive, des sensations de différents genres peuvent fusionner ensemble. Pour Stumpf, comme nous allons le voir, le phénomène de la fusion ne peut être subdivisé de cette manière, bien que nous puissions parler de différents degrés de fusion. De plus, Stumpf reconnaît la fusion entre différents sens. Alors que Wundt, considérant la fusion dans le cas de l'assimilation et de la reproduction ${ }^{54}$, affirme que des représentations multiples fusionnent ensemble en une seule nouvelle représentation, Stumpf affirmera seulement que de telles présentations sont "moins faciles à reconnaître et sont complètement reconnues comme une pluralité ${ }^{55}$.

Dans la troisième édition de Grundzüge der physiologischen Psychologie, Wundt s'est référé au travail de Carl Lorenz, qui avait mené une série d'expérimentations concernant les intervalles tonaux ${ }^{56}$ et largement discuté (et critiqué à l'occasion) l'approche de Stumpf dans Tonpsychologie. Selon Lorenz, les résultats de Stumpf sont douteux en raison des circonstances de ses expérimentations et particulièrement en raison de l'échantillon très réduit. En tentant de mesurer quel son est perçu comme le son médian entre deux autres, Lorenz avait découvert que la façon d'y parvenir était plus près de l'arithmétique que de la géométrie. Ce n'est pas la proportion relative (musicale), mais la fréquence absolue qui serait déterminante pour la sensation des sons médians, c'est-à-dire que le point médian de la sensation (Empfindungsmitte) serait plus près du point médian du stimulus (Reizmitte) ${ }^{57}$.

Stumpf répondit par une recension courte et négative puis rédigea un article détaillé sur le même sujet ${ }^{58}$, comprenant une défense de sa propre position et un nombre d'attaques à peine voilées à l'adresse de Wundt, répétant en partie des critiques plus anciennes. Il s'ensuivit une rude controverse

52. Wundt, 1880, p. 10.

53. Ibid., p. 13.

54. Voir la citation des Grundzüge plus haut, et Wundt, 1880, p. 15.

55. Stumpf, 1883, p. 101.

56. Publié plus tard dans Lorenz, 1890.

57. Lorenz, 1890, pp. 49, 64, 87, 99.

58. Stumpf, $1890 a$, Stumpf, $1890 c$. 
entre Stumpf et Wundt ${ }^{59}$, l'un et l'autre s'accusant d'être non scientifiques, de mener des attaques ad hominem et de ne pas vraiment comprendre les problèmes en cause ${ }^{60}$.

D'autre part, Stumpf se disait plutôt près de l'usage du concept de fusion fait par Ernst Heinrich Weber: "cette relation particulière de sensations simultanées est ce que $\mathrm{E}$. H. Weber a nommé le mélange ou la fusion ${ }^{61}$. Toutefois, Weber n'a pas développé systématiquement le type de conception philosophico-psychologique de la fusion (tonale) comme l'a fait Stumpf.

Dans les recherches de Stumpf sur les phénomènes tonaux, sa méthodologie est sensiblement différente de celle de Helmholtz ${ }^{62}$ puisqu'il suit la méthode de psychologie descriptive de Brentano au lieu de l'approche génétique, psycho-physique et physiologique. Pour Helmholtz, la consonance et la dissonance sont basées sur les caractéristiques physiques des sons euxmêmes considérés comme phénomènes naturels, et sur les propriétés physiologiques des nerfs et de l'œil humain. Pour Stumpf, par contre, les aspects les plus importants sont à trouver dans la manière dont nous percevons et expérimentons consciemment les sons, c'est-à-dire dans le fait qu'entendre des sons, les sons entendus étant des phénomènes mentaux. La consonance et la dissonance dépendraient encore ici des sons, mais en tant que ceux-ci sont considérés comme des contenus mentaux et non comme des phénomènes physiques externes. Ainsi, à la place de la causalité naturelle et des lois physiques, les lois psychologiques jouent un rôle important, car les actes mentaux et leurs contraintes (l'attention) influencent les phénomènes de consonance et de dissonance. Dans le contraste marqué avec Helmholtz, Stumpf caractérise le concept de fusion au chapitre VI de Tonpsychologie de la manière suivante:

La consonance de deux sons ne repose pas [...] sur les harmoniques ou sur des causes autres que les sons consonants eux-mêmes, mais sur une relation sensible particulière de ceux-ci entre eux, selon laquelle ils sont reconnus comme pluralité moins facilement et moins parfaitement que les sons dissonants. On ne peut concevoir d'explication physiologique, sans même parler de l'apporter comme preuve, pour cette relation que nous appelons fusion à défaut de meilleure expression, là où des deux sons ne résulte aucunement un son médian $^{63}$.

Les sons consonants ne sont pas perçus comme multiplicité, mais comme un type particulier de tout unitaire. Nous savons cependant que ce tout a été généré par une multiplicité et pouvons donc conclure que cette

59. Wundt, 1890: Stumpf, $1891 b$ : Wundt, $1892 b$.

60. Stumpf, 1891a' Wundt, $1892 a$.

61. Stumpf, 1890 b, p. $64 f$.

62. Dans Helmholtz, 1863.

63. Stumpf, 1883, p. 101. Ce passage a été souligné par Husserl dans son propre exemplaire, conservé aux Archives Husserl sous la signature BQ 472. 
multiplicité fusionne en un tout. Les sons fusionnés ne sont pas perçus comme des sons individuels nouveaux, ou des sons médians, mais comme un nouveau tout qui est basé sur une relation particulière entre les sons originaux. Stumpf a aussi décrit la fusion de cette manière dans ses leçons de psychologie:

On pourrait parler d'une fusion des deux sons. Mais on ne doit pas penser par là que les deux sons se fusionneraient en un son médian. Do et $m i$ restent ce qu'ils sont, mais l'impression générale se rapproche de celle d'un son unitaire ${ }^{64}$.

Encore une fois, Stumpf confirme que les deux sons fusionnés ne donnent pas un son médian, mais que l'impression est celle d'un nouveau tout, lequel est perçu comme un son et ne se décompose pas en une multiplicité.

Dans Tonpsychologie II, Stumpf se penche plus à fond sur la fusion. Il distingue plus clairement entre l'unité des contenus fusionnés et l'unité d'un collectivum. Avec la fusion, les qualités sensorielles apparaissent en fait comme une partie d'un tout sensoriel:

Les qualités ne sont pas modifiées le moins du monde, sans même parler du fait qu'elles ne sont pas transformées en une seule nouvelle qualité, mais une nouvelle relation entre elles fait son apparition, qui produit une unité plus étroite que celle qui a lieu entre les membres d'une simple somme ${ }^{65}$.

Dans une note de bas de page, Stumpf se réfère explicitement à l'Habilitationsschrift de Husserl pour la caractérisation des sommes comme des composites formés à partir de contenus complètement hétérogènes. Husserl, à son tour, cite précisément ce passage de Stumpf dans $P A^{66}$. Stumpf caractérise la fusion sur la base de la reconnaissance moins parfaite de la multiplicité d'éléments, mais la distingue d'autres cas. Je ne peux me représenter la couleur sans l'extension, ou vice versa, mais « les parties [d'un tout fusionné] ne sont pas, comme dans ce cas, inséparables [comme dans le cas d'un tout métaphysique, par exemple la couleur et l'extension] ${ }^{67}$. Ainsi, que je perçoive toujours les sons comme fusionnés, ce n'est pas là une propriété qui leur est nécessaire. La simultanéité des contenus semble être fondamentale à la fusion selon Stumpf: « Les sensations subséquentes constituent comme sensations une simple somme; les sensations simultanées, comme sensations, un tout ${ }^{68}$.»

Tout comme Husserl, Stumpf parle de différents degrés de fusion. Je reviendrai sur cela un peu plus loin. Mais penchons-nous maintenant sur l'usage que Husserl fait du concept de fusion dans Philosophie der Arithmetik.

64. Stumpf, WS 1886-1887a, p. 192.

65. Stumpf, $1890 b$, p. 64.

66. Husserl, 1891, p. 231; Husserl, 1970, p. 206; Husserl, 2003, p. 218.

67. Stumpf, $1890 b$, p. 65.

68. Stumpf, $1890 b$, p. 64. 
Husserl se réfère explicitement à Stumpf comme ayant inspiré son usage du concept de fusion ${ }^{69}$. Bien que Husserl emploie ce concept de manière particulière, des doutes ont été soulevés dans la littérature secondaire quant au fait que le concept de fusion de Stumpf et de Husserl soit vraiment le même. Je vais ici formuler les objections et montrer ensuite que, en comparaison avec des différences beaucoup plus radicales, par exemple celles entre Herbart et Wundt, les concepts de fusion de Stumpf et Husserl peuvent être considérés comme le même concept sous leurs aspects les plus importants.

Dans sa discussion des moments figuraux, Husserl fait remarquer qu'une sorte de fusion a lieu sur le plan des relations entre les éléments d'une quantité qui a un moment figural. Avec des quantités comme un tas de pommes, une rangée de soldats, un troupeau de vaches, etc., nous avons des représentations de multiplicités d'objets identiques. Pour pouvoir appréhender ces quantités comme quantités de manière immédiate et non par dénombrement, certaines quasi-qualités doivent être données dans l'intuition: ce sont les moments figuraux, les Gestaltmomente.

Nous allons en effet tenter de justifier la conception selon laquelle ces moments [figuraux - moments de Gestalt] doivent être considérés justement comme unités dans lesquelles les particularités des contenus ou de leurs relations primaires fusionnent les uns avec les autres. Je dis ici «fusionnent ", et je veux par là mettre l'accent sur le fait que les moments unitaires sont quelque chose d'autre que de simples sommes. Nous saisissons le caractère quasi-qualitatif de l'intuition complète comme quelque chose de simple, et non comme un collectivum de contenus et de relations ${ }^{70}$.

Tout comme Stumpf, Husserl souligne la distinction fondamentale entre les collectiva et les touts fusionnés. Un collectivum est toujours perçu comme une multiplicité, une somme, alors que dans le cas de la fusion une quantité est appréhendée comme un tout unitaire de sensations.

L'approche de Husserl peut nous rappeler la discussion de l' «agglutination» chez Wundt:

Les représentations complètes sont toujours à la fois des actes de pensée individuels, donc des connexions simultanées, qui toutefois, selon le contexte, comme c'est le cas avec les agglutinations, se produisent seulement après le défilement d'une pluralité de représentations individuelles ${ }^{71}$.

Cependant, la conception de Wundt fait abstraction de la distinction faite dans l'école de Brentano entre les représentations propres et les représentations impropres. La description de Wundt peut être considérée comme similaire à la conception husserlienne des représentations propres de nombre et de quantité, mais Husserl emploie la fusion dans Philosophie der Arithmetik

69. Husserl, 1891, p. 228f.; Husserl, 1970, p. 204; Husserl, 2003, p. 216.

70. Husserl, 1891, p. 228sq.; Husserl, 1970, p. 204; Husserl, 2003, p. 216.

71. Wundt, 1880, p. 28. 
précisément pour expliquer la possibilité de représentations impropres, c'est-à-dire symboliques, de nombres et de quantités. Husserl s'est donné beaucoup de mal sur la question de l'appréhension immédiate des quantités comme quantités, c'est-à-dire une appréhension qui ne requiert précisément pas d'énumération (exhaustive) de ses membres. En effet, la Verschmelzung et les qualités de forme qui en résultent sont préalables pour une telle analyse et pour l'énumération.

Une multiplicité d'objets de la même sorte est appréhendée comme quantité d'un type particulier. Elle est perçue comme quelque chose de simple et non comme un complexe: comme un tout, et non comme une somme. Comment cela est-il possible? Les objets et les relations entre ceux-ci fusionnent ensemble et donnent un tout d'ordre supérieur. Une quantité de soldats forme une armée, une quantité de vaches un troupeau, une quantité de pommes un tas de pommes, etc. Lorsque j'observe ces quantités, je peux immédiatement les reconnaître comme quantités, sans avoir à parcourir une longue série de comparaisons et d'additions que je ne pourrais probablement même pas compléter ${ }^{72}$.

Mis à part les relations d'égalité de premier ordre, c'est-à-dire entre les pommes d'un tas de pommes, nous notons également que toutes ces relations d'égalité sont les mêmes, c'est-à-dire qu'elles sont toutes de la même sorte de relation d'égalité. Vus de cette perspective d'ordre supérieur, les contenus et les relations primaires fusionnent et constituent un tout d'ordre supérieur, comme une armée, un troupeau, un tas, etc. La relation d'égalité de second ordre est cruciale entre les relations d'égalité de premier ordre. C'est précisément le type de relation que Husserl cherchait: "un caractère particulier immédiatement remarquable, une qualité sensible de deuxième ordre, pour ainsi dire ${ }^{73}$.

Penchons-nous maintenant sur les objections à la thèse selon laquelle les conceptions de la Verschmelzung de Husserl et de Stumpf seraient la même. Elmar Holenstein et Robin Rollinger soutiennent que la conception husserlienne de la fusion est différente de la conception stumpfienne. Holenstein explique:

Alors que, pour Stumpf, la fusion ne peut être déduite de la similarité des éléments entre eux, Husserl considère la similitude sensible comme une des propriétés caractéristiques qui contribuent à la formation d'une unité ${ }^{74}$.

Rollinger élabore cet argument plus en détail. C’est parce que Husserl fonde la fusion sur les quasi-qualités, qui font un tout fusionné en partant d'une simple collection, que le collectivum et le tout fusionné ne seraient pas mutuellement exclusifs: "Pour que les contenus soient fusionnés, ils doivent

72. Husserl, 1891, p. 233; Husserl, 1970, p. 208; Husserl, 2003, p. 220.

73. Husserl, 1891, p. 225; Husserl, 1970, p. 201; Husserl, 2003, p. 213.

74. Holenstein, 1972, p. 109. 
être représentés ensemble dans une collection ${ }^{75}$.» Tous les touts fusionnés sont donc toujours un collectivum, mais considéré seulement de manière impropre et momentanée. La raison motivant l'introduction des quasi-qualités dans la $P A$ était de garantir la propriété d'être une quantité lorsqu'on ne peut appréhender chacun des membres dans une succession et construire une collection. Néanmoins, une quantité peut être appréhendée comme quantité, car le caractère de quantité peut être reconnu grâce aux moments figuraux, les quasi-qualités. Dans le cas de Stumpf, toutefois, les sensations appréhendées successivement et simultanément s'excluent l'une et l'autre. Conséquemment, affirme Rollinger, le concept de fusion de Stumpf est différent de celui de Husserl.

Bien que ces objections soulèvent des questions importantes sur la compatibilité des conceptions de Husserl et de Stumpf, je crois que la thèse de l'exclusion mutuelle des sensations appréhendées successivement et simultanément est trop forte. N'importe quelle sensation simultanée peut toujours être appréhendée successivement lorsqu'on dirige son attention d'abord sur une d'entre elles, puis sur une autre, etc. Stumpf affirme dans ses leçons:

L'attention est un fonds qui a un certain volume, lequel diminue lorsqu'il est distribué parmi ceux qui y ont droit. Si on essaie de distribuer son attention sur plusieurs choses en même temps, celle-ci devient en fin de compte pratiquement nulle. Et si on essaie de concentrer un maximum d'attention sur des représentations, ce n'est alors que sur une représentation, ou quelques-unes ${ }^{76}$.

Dès que je porte mon attention sur des éléments singuliers donnés du tout de sensations, cela échoue, car je ne peux retenir toutes les impressions de ma perception complète à la fois comme tout et comme séparé en même temps. De manière analogue, n'importe quel tout fusionné peut être analysé et sujet à une appréhension successive. C'est seulement lorsque le nombre d'éléments qui doivent être successivement appréhendés dépasse ma capacité de représentation que je dois croire aveuglément aux quasi-qualités pour établir la nature d'une quantité.

Il est possible de considérer successivement des touts d'impressions simultanées, un par un, pour tous les sens. Je vois une salle remplie de monde et je détermine d'un coup d'œil: une foule de gens, c'est-à-dire une quantité (dénombrable) de personnes ${ }^{77}$. Ainsi, j'ai visiblement la possibilité de dénombrer les éléments individuels du tout. Je dirige successivement mon attention sur les différents éléments de la quantité et les constitue en un collectivum. Cependant, avec les grandes quantités, on ne peut retenir le tout de sensations d'une «salle pleine de monde» dans l'intuition et remarquer simultanément

75. Rollinger, 1999, p. 109.

76. Stumpf, WS, 1886-1887b, p. 611.

77. En allemand, «eine Menge» veut dire aussi bien «une quantité» qu'« une multitude». 
tout un chacun dans la salle, puis l'ajouter à l'ensemble. C'est seulement en ce sens que le collectivum et les touts fusionnés ne peuvent coexister. Sur ce point, Rollinger pourrait éventuellement rétorquer que cette variété affaiblie d'exclusion serait tout ce qui est nécessaire pour distinguer le concept de fusion de Stumpf et celui de Husserl. Cependant, à mon avis, la distinction n'est pas si fondamentale qu'elle le paraît.

Stumpf semble nier que l'attention puisse jouer un rôle dans la détection de sons individuels dans un tout fusionné:

Si deux sons avec des fréquences oscillatoires dans des rapports de 1:2 sont produits simultanément, ils ne peuvent être isolés que de manière très imparfaite [...]. Par «imparfait", je veux dire qu'il ne s'agit pas d'une simple difficulté qui peut être contournée en augmentant l'attention et l'exercice ${ }^{78}$.

Néanmoins, comme on l'a vu dans les citations mentionnées plus haut, les sons ne se fondent pas véritablement dans un "son médian", ils ne deviennent pas un seul son, mais ils s'apparentent à la perception d'un son individuel, c'est-à-dire que Stumpf distingue le fait d'entendre un son individuel et le fait d'entendre une multiplicité de sons comme s'ils étaient un seul son: «le caractère d'unité n'est pas l'unité », comme Stumpf le soulignera plus loin:

Même lorsque nous reconnaissons les sons comme deux sons et que nous les distinguons, ils constituent toutefois un tout dans la sensation, et ce tout nous semble avoir parfois plus, parfois moins d'unitée ${ }^{79}$.

Le caractère d'unité n'est pas l'unité. La fusion ne signifie pas qu'on ne distinguerait aucunement entre les deux sons ${ }^{80}$.

Ainsi, la fusion ne disparaît aucunement, mais l'exercice et l'attention donnent à l'auditeur la possibilité de reconnaître la multiplicité sous-jacente comme telle, malgré la fusion. "Ceux qui ont l'oreille musicale distinguent constamment les sons dans les cas habituels ${ }^{81}$.» Si l'attention et l'exercice étaient effectivement complètement inutiles, on pourrait fermer demain tous les conservatoires. En fin de compte, Stumpf lui-même est préoccupé par le fait d'entendre différentes composantes de sons et des sons partiels à même des complexes de sons ${ }^{82}$. Dans les citations que j'ai présentées ici, Stumpf fournit de manière succincte ce qui est nécessaire pour formuler l'objection: les sons peuvent être reconnus comme une multiplicité tout en étant fusionnés. La fusion de contenus simultanés n'exclut pas une analyse passant par le dénombrement.

78. Stumpf, $1890 b$, p. 127.

79. Stumpf, 1898, p. 35.

80. Stumpf, 1911, p. 118.

81. Stumpf, 1911, p. 118.

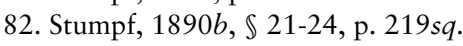


Même les commentaires très épars de Brentano sur la fusion dans Psychologie vom empirischen Standpunkte suffisent pour clarifier ce point ${ }^{83}$ :

Car il arrive que plusieurs représentations fusionnent en une seule de telle sorte qu'elles ne se présentent plus comme plusieurs, mais plutôt comme une seule représentation d'une tout autre nature ${ }^{84}$.

Toute fusion est une association du multiple, et, du coup, l'expression imagée $[\ldots]$ indique encore une certaine équivoque dans l'unitéess.

Les représentations ne fusionnent pas en une unité inextricable, comme Wundt le soutenait, mais elles se présentent comme fusionnées. La multiplicité ne disparaît pas complètement, les contenus demeurent où ils sont, mais ils se présentent comme quelque chose de différent, c'est-à-dire comme un tout. Des sons perçus simultanément ne peuvent être appréhendés comme des sons successifs, mais ils peuvent être appréhendés séparément, alors que Stumpf (par erreur) semble définir les collectiva et les touts fusionnés de sensations sur cette base comme des classes complètement séparées. La distinction entre les collectiva et les fusions est basée sur une distinction d'appréhension: successive ou simultanée. Dans le cas de la vue, on peut aisément et spontanément exercer un contrôle explicite et conscient quant à savoir si une certaine quantité est appréhendée comme un tout en embrassant du regard tous les membres, ou au contraire on peut sélectionner chaque élément un par un et les mener successivement à une collection. Avec les sensations auditives on a moins de contrôle parce que, pour entendre les composantes séparées d'une tierce, par exemple, l'attention et l'exercice sont nécessaires. Je peux exercer le contrôle conscient et explicite nécessaires pour les distinguer et influencer ainsi l'appréhension de manière aussi simple et spontanée que pour la vision seulement lorsque je produis moi-même les sons. Lorsque je produis les sons simultanément ils fusionnent, et lorsque je les produis successivement, ils ne fusionnent pas. Stumpf dit en effet: "c'est seulement $s$ 'ils sont perçus simultanément qu'il est alors impossible de ne pas en avoir la sensation comme d'un tout, dans une relation de fusion ${ }^{86}$. Lorsque Stumpf affirme d'une part qu'il serait difficile d'entendre des octaves comme deux sons différents, car «ils ne peuvent être séparés que de manière très imparfaite ", et que d'autre part il affirme que, "s'ils sont perçus simultanément, il est alors impossible de ne pas en avoir la sensation comme d'un tout dans une relation de fusion ", cela implique déjà, comme le confirment

83. Brentano parle également de cas de fusion d'actes et de la fusion de l'acte avec son objet dans un contexte différent lorsqu'il traite de Mill et d'Hamilton. Brentano n'a pas développé une théorie complète de la fusion dans Psychologie...

84. Brentano, 1874, p. 15sq.

85. Ibid., 118.

86. Stumpf, $1890 b$, p. 65 . Ce passage a été souligné par Husserl dans son propre exemplaire par un «NB» dans la marge. 
les citations des ouvrages plus tardifs, qu'on peut en faire l'expérience simultanément comme étant fusionnés et séparés. Comme Husserl l'affirme quant au concept propre d'Inbegriff: «La complication de ces processus, progressant de manière extrêmement rapide, exige un effort énorme pour notre capacité représentationnelle ${ }^{87}$.» Cette capacité peut être précisément augmentée par "une attention et un exercice renforcés». Et cela est aussi vrai pour les autres sens: lorsque les sensations sont données en même temps, elles peuvent fusionner, et lorsqu'elles ne le sont pas, nous pouvons les appréhender dans une succession. En écoutant, l'auditeur n'a qu'un contrôle réduit sur le genre d'appréhension, par simultanéité ou par succession, mais avec d'autres sens un contrôle accru est possible. Et puisque Stumpf admet la fusion pour tous les sens, et même entre différents sens ${ }^{88}$, nous ne pouvons baser une caractérisation générale de la fusion pour tous les sens seulement sur le cas exceptionnel de l'audition. Puisqu'on parle toujours d'un certain degré de fusion lors de l'audition simultanée de deux ou plusieurs sons (même dans le cas de sons absolument dissonants), on ne peut simplement affirmer sans plus d'investigation que toutes les impressions simultanées doivent nécessairement toujours être fusionnées aussi bien par les sens qu'à travers eux. De plus, lorsqu'on considère que Husserl et Stumpf parlent tous deux de différents degrés de fusion, on peut se demander ce qui détermine ces degrés différents.

Stumpf affirme: «Les sensations simultanées d'un seul sens fusionnent à un degré plus important que les différents sens ${ }^{89}$. J'ai tendance à penser que cela est dû à la similarité ou même à l'égalité au sein des sens. Les sensations visuelles sont davantage similaires les unes aux autres qu'une sensation visuelle et une sensation olfactive. La similarité n'est en fait rien d'autre que l'égalité partielle, et il est évident que deux sensations visuelles ont davantage d'égalité partielle qu'une sensation visuelle et une sensation olfactive (considérons par exemple la couleur et la spatialité dans la sensation visuelle). Stumpf discute précisément de ce problème dans le $\mathbb{2 0 . 2}$ de Tonpsychologie II:

Plus les sensations sont similaires, plus elles fusionnent avec un degré élevé d'intensité. Mais cette remarque n'est pas contraignante, car la similarité et la fusion peuvent être dépendantes d'un troisième facteur commun ${ }^{90}$.

La similarité et la fusion semblent varier au même degré, et on pourrait donc supposer, comme le fait Stumpf, que la similarité et la fusion ont une cause commune, puisqu'il refuse de dire que la similarité est la base de la fusion ${ }^{91}$.

87. Husserl, 1891, p. 55; Husserl, 1970, p. 54; Husserl, 2003, p. 55.

88. Stumpf, 1890 b, p. $65 f$.

89. Ibid., p. 65.

90. Ibid., p. 193.

91. Ibid., p. 204. 
Quelles options avons-nous alors? À part la similarité, la consonance varie aussi au même degré que la fusion. Les sons qui sont le plus consonants, comme les octaves, sont les plus fusionnés. Après les octaves, ce sont les quintes, les quartes, les tierces majeures, etc., qui décroissent dans la fusion. Moins les sons sont consonants, moins ils fusionnent ${ }^{92}$. La consonance est si étroitement liée à la fusion que Stumpf va même jusqu'à les identifier ${ }^{93}$. Cependant, il n'a encore expliqué nulle part ce qu'est cette "relation sensible particulière", "en raison de laquelle [les sons consonants] sont moins facilement et moins parfaitement reconnus comme multiplicité que les sons dissonants ${ }^{94}$.

En conclusion, et à défaut d'une meilleure explication, Stumpf conclut que la fusion doit être expliquée physiologiquement, puisque toutes les tentatives psychologiques ont échoué ${ }^{95}$. Malheureusement, il doit concéder que, dans ce cas, il n’y a pas grand chose à en dire, mais il préfère néanmoins cette "honnête pauvreté » ${ }^{96}$.

Selon Husserl, comme nous l'avons vu, non seulement les relations d'égalité de premier ordre, mais aussi et surtout les relations de second ordre, jouent un rôle important. Or, dans le cas de Stumpf, il y a aussi un exemple qui semble tenir compte des relations d'égalité de second ordre:

Lorsque, dans une harmonie consonante dont les membres fusionnent à un degré élevé (il s'agit ici de membres très nombreux), intervient un seul son dissonant de même intensité que les autres (et fusionné avec les autres au niveau le plus bas), on remarque alors très facilement la différence ${ }^{97}$.

Or la différence qu'on remarque très facilement dans ce cas est une différence de second ordre. On remarque en fait qu'il y a une différence entre les relations subsistant entre les sons consonants et la relation du nouveau son avec ceux-ci. On remarque ainsi une relation de différence entre d'autres relations. Et alors, on peut bien entendu aussi parler d'une relation d'égalité de second ordre dans le premier cas, là où il n'y a pas encore de son dissonant, parce que tous les sons ont de manière évidente la même relation les uns aux autres. Ainsi, un tout fusionné doit être basé sur la reconnaissance de l'égalité de la relation de consonance. Ce que la similarité a fait pour la vision chez Husserl, la consonance le fait pour l'audition chez Stumpf. Cela semble beaucoup moins controversé si nous prenons la liste des relations fondamentales de Stumpf dans Tonpsychologie I comme une hiérarchie de degrés plus ou moins élevés d'égalité construits les uns sur les autres ${ }^{98}$.

92. Stumpf, 1890 b, p. 371.

93. "J'identifie déjà ici la consonance avec les niveaux supérieurs de fusion, et la dissonance avec les niveaux les plus inférieurs ", Stumpf, 1890b, p. 333n.

94. Stumpf, 1883, p. 101.

95. Stumpf, $1890 b, \$ 6$, p. 211.

96. Ibid., p. 214.

97. Ibid., p. 332sq.

98. Stumpf, 1883, p. 96sq. Pour cette conception, voir Schneider, 1997. 
Ce qu'apporte la fusion entre différents sens, c'est la relation d'égalité de second ordre, et pas seulement un type de relation particulière de premier ordre. Rollinger et Holenstein ont raison lorsqu'ils affirment que chez Stumpf ce n'est pas la similarité qui cause la fusion, mais c'est également le cas chez Husserl: ce n'est pas une relation de similarité de premier ordre, mais une relation d'égalité de second ordre. Pour chacun de nos sens, il y a probablement une relation particulière de premier ordre telle que la similarité ou la consonance, qui ne peut qu'indirectement conduire la fusion vers un tout. L'élément principal est la relation de second ordre. Nous l'avons trouvée chez Husserl et chez Stumpf et pouvons conclure que leur concept de fusion est le même, eu égard à leurs aspects importants. Lorsque Stumpf rejette la similarité, il le fait dans le contexte de la musique. Toutefois, la fusion ne touche pas uniquement le domaine de l'audition mais également d'autres sens, et, comme Stumpf le dit, elle a lieu même entre les sens ${ }^{99}$. Ce dernier cas (la fusion entre les sens) requerrait probablement une autre relation d'ordre encore plus élevé, mais ce n'est pas ici le lieu pour poursuivre cette investigation.

Traduit de l'anglais par Guillaume Fréchette

\section{Bibliographie}

Bolzano, Bernard. 1889. Paradoxien des Unendlichen, Berlin, Mayer \& Müller.

Brentano, Franz. 1874. Psychologie vom empirischen Standpunkt, Leipzig, Duncker \& Humblot.

Ehrenfels, Christian von. 1890. «Uber Gestaltqualitäten ». In Vierteljahrsschrift für wissenschaftliche Philosophie 14. Trad. fr. dans D. Fisette, G. Fréchette (dir.), À l'école de Brentano. De Würzburg à Vienne, Paris, Vrin, 2007.

Gerlach, Hans-Martin et Hans Rainer Sepp (dir.). Husserl in Halle. Daedalus 5 (Europäisches Denken in Deutscher Philosophie), Francfort-sur-le-Main, Peter Lang GmbH, 1994.

Helmholtz, Hermann von. 1863. Die Lehre von den Tonempfindungen als physiologische Grundlage für die Theorie der Musik, Braunschweig, Vieweg und Sohn.

Herbart, Johann Friedrich. 1825. Psychologie als Wissenschaft: Neu gegründet auf Erfahrung, Metaphysik und Mathematik, Königsberg, Unzer.

-. 1851. Sämmtliche Werke. Gustav Hartenstein (dir.), Leipzig, Voss.

Holenstein, Elmar. Phänomenologie der Assoziation. Phaenomenologica 44, La Haye, Nijhoff, 1972.

Hopkins, Burt. "Authentic and Symbolic Numbers in Husserl's Philosophy of Arithmetic». In The New Yearbook for Phenomenology and Phenomenological Philosophy II, 2002, pp. 39-71.

Husserl, Edmund. 1887. Über den Begriff der Zabl (Psychologische Analysen), F. Beyer (dir.), Halle a.d. Saale, Heynemann'sche Buchdruckerei. 
- 1891. Philosophie der Arithmetik (Psychologische und Logische Untersuchungen), Robert Stricker (dir.), Halle-Saale, C.E.M. Pfeffer.

—. Philosophie der Arithmetik. Lothar Eley (dir.). Husserliana XII, La Haye, Nijhoff, 1970.

- Logische Untersuchungen (Zweiter Band, Erster Teil). Ursula Panzer (dir.). Husserliana XIX/1, La Haye, Nijhoff/Kluwer, 1984.

_. Briefwechsel. Karl Schuhmann et Elisabeth Schuhmann (dir.). Husserliana Dokumente III, Dordrecht, Kluwer, 1994.

—. Logische Untersuchungen (Ergänzungsband: Erster Teil). Ullrich Melle (dir.), Husserliana XX/1, Dordrecht, Kluwer, 2002.

- Philosophy of Arithmetic. Trad. angl. de Dallas Willard. Edmund Husserl Collected Works X, Dordrecht, Kluwer, 2003.

—. «Lecture On the Concept of Number (WS 1889-1890)». Trad. de Carlo Ierna. In The New Yearbook for Phenomenology and Phenomenological Philosophy V., 2005a, 279-309 impair.

—. "Vorlesung Über den Begriff der Zahl (WS 1889-1890)». Carlo Ierna (dir.). In The New Yearbook for Phenomenology and Phenomenological Philosophy V. 2005b, 278-308 pair.

Ierna, Carlo. "The Beginnings of Husserl's Philosophy. Part 1: From Über den Begriff der Zahl to Philosophie der Arithmetik». In The New Yearbook for Phenomenology and Phenomenological Philosophy V., 2005, pp. 1-56.

—. "The Beginnings of Husserl's Philosophy. Part 2: Mathematical and Philosophical Background». In The New Yearbook for Phenomenology and Phenomenological Philosophy VI, 2006, pp. 23-71.

Lorenz, Carl. 1890. «Untersuchungen über die Auffassung von Tondistanzen». In Wundts Philosophische Studien VI.1, pp. 26-103.

Mach, Ernst. 1886. Beiträge zur Analyse der Empfindungen. Jena, Gustav Fischer.

- The Analysis of Sensations and the Relation of the Physical to the Psychical. Traduction anglaise de Williams et Waterlow, Chicago/Londres, Open Court, 1914.

Meinong, Alexius. 1882. "Hume Studien II. Zur Relationstheorie». In Sitzungsbereiche der phil.-hist. Classe der kais. Akademie der Wissenschaften CI.II, pp. 573-752.

Mulligan, Kevin. «Perception». In The Cambridge Companion to Husserl, Barry Smith et David Woodruff Smith (dir.), Cambridge, Cambridge University Press, 1995.

Petitot, Jean. "Morphological Eidetics for a Phenomenology of Perception". In Naturalizing Phenomenology, J. Petitot et al. (dir.), Stanford, Stanford University Press, 1999.

Rollinger, Robin. Husserl's Position in the School of Brentano, Phaenomenologica 150, Dordrecht, Kluwer, 1999.

Schneider, Albrecht. "'Verschmelzung'. Tonal Fusion, and Consonance: Carl Stumpf Revisited». In Marc Leman (dir.), Music, Gestalt, and Computing - Studies in Cognitive and Systematic Musicology, vol. 1317. Lecture notes in computer science, Berlin/New York, Springer, 1997, pp. 117-143.

Schuhmann, Karl. Husserl: Chronik (Denk- und Lebensweg Edmund Husserls). Husserliana Dokumente I, La Haye, Nijhoff, 1977.

Smith, Barry (dir.). Foundations of Gestalt Theory, Munich, Philosophia, 1988a. 
—. "Gestalt Theory: An Essay in Philosophy». In Foundations of Gestalt Theory, Barry Smith (dir.), Munich, Philosophia, 1988b, pp. 11-80.

—. Austrian Philosophy, Chicago, Open Court, 1994.

Stumpf, Carl. 1873. Über den Psychologischen Ursprung der Raumvorstellung, Leipzig, Hirzel.

—. 1883. Tonpsychologie, vol. 1, Leipzig, Hirzel.

-1890a. «Rezension von C. Lorenz: Untersuchungen über die Auffassung von Tondistanzen ». In Zeitschrift für Psychologie und Physiologie der Sinnesorgane 1.

—. 1890b. Tonpsychologie, vol. 2, Leipzig, Hirzel.

-1890c. "Über Vergleichungen von Tondistanzen ». In Zeitschrift für Psychologie und Physiologie der Sinnesorgane 1, pp. 419-462.

—. 1891a. "Mein Schlußwort gegen Wundt». In Zeitschrift für Psychologie und Physiologie der Sinnesorgane 2, pp. 438-443.

—. 1891b. "Wundts Antikritik». In Zeitschrift für Psychologie und Physiologie der Sinnesorgane 2, pp. 266-293.

—. 1898. "Konsonanz und Dissonanz». In Beiträge zur Akustik und Musikwissenschaft I, pp. 1-108.

—. 1911. «Konsonanz und Konkordanz. Nebst Bemerkungen über Wohlklang und Wohlgefälligkeit musikalischer Zusammenklänge ». In Beiträge zur Akustik und Musikwissenschaft 6, pp. 116-150.

—. (SS 1887). Q 14: Logik und Enzyklopädie der Philosophie. (Notes de Husserl des leçons de Stumpf, SS 1887. Transcription de E. Schuhmann et K. Schuhmann), Halle.

—. (WS 1886-1887a). Ms. Q 11/I: Vorlesungen über Psychologie. (Notes de Husserl des leçons de Stumpf, WS 1886-1887. Transcription de E. Schuhmann et K. Schuhmann, basée sur une transcription partielle de R. Rollinger).

—. (WS 1886-1887b). Ms. Q 11/II: Vorlesungen über Psychologie. (Notes de Husserl des leçons de Stumpf, WS 1886-1887. Transcription de E. Schuhmann et K. Schuhmann, basée sur une transcription partielle de R. Rollinger).

Wundt, Wilhelm. 1874. Grundzüge der physiologischen Psychologie, Leipzig, Engelmann.

- 1880. Logik. Eine Untersuchung der Principien der Erkenntniss und der Methoden wissenschaftlicher Forschung. 1 $1^{\text {re }}$ éd., vol. 1. Erkenntnisslehre, Stuttgart, Enke.

—. 1890. «Über Vergleichungen von Tondistanzen ». In Philosophische Studien 6, pp. 605-640.

—. 1892a. "Auch ein Schlusswort» In Philosophische Studien 7, pp. 633-636.

—. 1892b. «Eine Replik C. Stumpf's». In Philosophische Studien 7, pp. 298-327. 